\title{
Serological and histological indices of hepatocellular carcinoma and tumor volume doubling time
}

\author{
NAOKI SHINGAKI, HIDEYUKI TAMAI, YOSHIYUKI MORI, KOSAKU MORIBATA, SHOTARO ENOMOTO, \\ HISANOBU DEGUCHI, KAZUKI UEDA, IZUMI INOUE, TAKAO MAEKITA, \\ MIKITAKA IGUCHI, JUN KATO and MASAO ICHINOSE
}

Second Department of Internal Medicine, Wakayama Medical University, Wakayama City, Wakayama 641-0012, Japan

Received June 14, 2013; Accepted August 29, 2013

DOI: $10.3892 / \mathrm{mco} .2013 .186$

\begin{abstract}
Hepatocellular carcinoma (HCC) frequently develops in cirrhotic liver and is one of the most common malignancies worldwide. The tumor volume doubling time (TVDT) reflects the natural tumor growth rate and is an indicator of the biological malignant potential of a tumor. The present study aimed to elucidate the association between the serological and histological indices of HCC and TVDT. TVDT was analyzed for 53 HCCs by measuring the enlargement of the tumor diameter on dynamic computed tomography. Differences in TVDT were compared among histological grades of HCC differentiation. The $\alpha$-fetoprotein (AFP) doubling time (T2AFP) for 44 HCCs with AFP levels $>200 \mathrm{ng} / \mathrm{ml}$ was calculated and the differences in T2AFP were compared according to the histological grade of HCC differentiation and positivity for Lens culinaris agglutinin-reactive fraction of $\alpha$-fetoprotein (AFP-L3). Among these $44 \mathrm{HCCs}$, the correlation between T2AFP and TVDT was analyzed for the 27 tumors for which TVDT could be calculated. The mean \pm standard deviation (SD) TVDT in Edmondson grade 1 (Ed1), Ed2 and Ed3 HCC was $138.3 \pm 110.3,94.9 \pm 91.5$ and $32.2 \pm 20.8$ days, respectively $(\mathrm{P}<0.05)$. The mean \pm SD T2AFP in Ed2 and Ed3 HCC was $121.0 \pm 167.5$ and $37.3 \pm 24.6$ days, respectively $(\mathrm{P}<0.01)$. TVDT and T2AFP decreased with histological dedifferentiation of HCC. The mean \pm SD T2AFP in the AFP-L3-positive

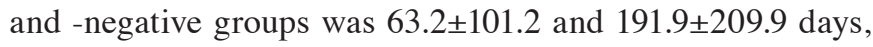
respectively, with a statistically significant difference between the groups $(\mathrm{P}<0.01)$. A significant correlation was observed between T2AFP and TVDT (correlation coefficient, 0.70; $\mathrm{P}<0.01)$. A significant correlation was also observed among TVDT, serological indices and histological grades of HCC differentiation. A short T2AFP and/or AFP-L3-positivity were
\end{abstract}

Correspondence to: Dr Hideyuki Tamai, Second Department of Internal Medicine, Wakayama Medical University, 811-1 Kimiidera, Wakayama City, Wakayama 641-0012, Japan

E-mail: tamahide@wakayama-med.ac.jp

Key words: $\alpha$-fetoprotein, $\alpha$-fetoprotein doubling time, hepatocellular carcinoma, histological differentiation, tumor volume doubling time shown to reflect a poorly differentiated HCC histopathology and a higher malignant potential.

\section{Introduction}

Hepatocellular carcinoma (HCC) frequently develops in cirrhotic liver and is one of the most common malignancies worldwide. HCC typically develops in a multistep fashion from a low-grade dysplastic nodule to advanced HCC (1). During the course of carcinogenesis, a steady stepwise histological dedifferentiation of the neoplastic cells occurs in conjunction with increasing malignant potential, leading from a well-differentiated HCC to a moderately and finally to a poorly differentiated HCC, with a significantly increased tendency for vascular invasion and metastasis (2). The prognosis of patients worsens with histological dedifferentiation of $\operatorname{HCC}(3,4)$.

The tumor volume doubling time (TVDT) reflects the natural tumor growth rate and is an indicator of the biological malignant potential of a tumor. The assessment of TVDT at the time of diagnosis provides a useful parameter for clinical decision making regarding the optimal therapeutic strategy and appropriate follow-up intervals. However, the calculation of TVDT requires values from multiple time points, making this value impossible to determine in numerous cases where treatment is initiated soon after diagnosis. To calculate TVDT, an obvious increase in tumor size must be detected by comparing two subsequent imaging examinations.

The serum $\alpha$-fetoprotein (AFP) level is one of the most commonly used tumor markers of HCC and is currently used for HCC surveillance and monitoring of recurrence after treatment. The serum levels of AFP and/or Lens culinaris agglutinin-reactive fraction of $\alpha$-fetoprotein (AFP-L3) are reportedly significantly higher in poorly differentiated HCC compared to those in well- or moderately differentiated HCC (5-7). Although AFP may increase non-specifically during the process of highly active hepatitis, the AFP doubling time (T2AFP) may reflect the tumor growth rate in AFP-producing HCCs. However, the correlation among TVDT, histological grade of differentiation and T2AFP has not been fully elucidated. If T2AFP correlates strongly with TVDT, the malignant potential of AFP-producing HCCs, which is reflected by the natural tumor growth rate, would be easily estimated, even over short intervals. 
The present study investigated the correlation among histological grade of HCC differentiation, TVDT and T2AFP, as well as between T2AFP and serum AFP-L3 levels, as an indicator of poor prognosis in $\mathrm{HCC}$.

\section{Patients and methods}

Patients. In order to compare TVDT by histological grade of HCC differentiation, based on our HCC database including patients admitted between April, 2001 and December, 2011, 53 patients with a histological diagnosis of HCC who met the following criteria were retrospectively identified: i) nodular type HCC; ii) availability of baseline and follow-up computed tomography (CT); iii) no treatment during the follow-up period; and iv) measurable enlargement of tumor diameter on dynamic $\mathrm{CT}$ during the waiting time prior to treatment.

To calculate T2AFP, based on our HCC database including patients admitted between April, 2001 and December, 2011, $44 \mathrm{HCC}$ patients who met the following criteria were identified: i) serum AFP level measured twice over a 14-day interval; ii) histologically confirmed diagnosis of HCC; iii) baseline serum AFP level $>200 \mathrm{ng} / \mathrm{ml}$, to exclude the possibility of non-specific AFP elevation (8); iv) follow-up serum AFP level higher than the baseline serum AFP level; and v) baseline serum AFP-L3 level measured. Furthermore, among the 44 HCC patients in whom T2AFP could be calculated, data from 27 HCC patients for whom TVDT could be calculated were analyzed to evaluate the correlation between T2AFP and TVDT. This retrospective study was approved by the Ethics Committee of Wakayama Medical University. The need for patient consent was waived by the Ethics Committee.

Histological diagnosis of HCC. For the evaluation of the histological grade of HCC differentiation, tissue samples were obtained from surgically resected specimens or needle biopsies of the above-described HCC patients. Tumor needle biopsy was performed using an 18-gauge core biopsy needle (Bard Monopty ${ }^{\circledR}$ no. 121820; Bard Biopsy Systems, Covington, GA, USA) under ultrasound guidance within 1 week prior to treatment. In patients with multiple HCC nodules, the largest nodule, which was presumed to affect prognosis, was assessed. Hematoxylin and eosin staining was performed on all the liver tumor specimens and histology was assessed by two pathologists who were blinded to the clinical course of the patients. Histological grading of HCC was performed according to the criteria described by Edmondson and Steiner (9). When heterogeneous histological grades of differentiation were observed in the obtained tissue sample, the highest grade was adopted. The fibrosis stage of the background liver was assessed using the METAVIR scoring system (10). In case of discrepancies between the evaluations of the two pathologists, a consensus was reached through discussion.

Dynamic CT. All contrast CT examinations were performed using multi-detector row CT scanners with at least 4 detectors (Aquilion; Toshiba Medical Systems, Tochigi, Japan, or LightSpeed VCT; GE Medical Systems, Milwaukee, WI, USA), with 5-mm sections. In addition to the plain images, arterial-phase images were obtained $40 \mathrm{sec}$ after the start of bolus administration of $580 \mathrm{mgI} / \mathrm{kg}$ of non-ionic iodinated contrast medium for $30 \mathrm{sec}$ via an automated power injector. From 2005 onward, the arterial phase was scanned using an automatic bolus-tracking program. Portal- and equilibrium-phase images were obtained at 70 and $180 \mathrm{sec}$ after the start of bolus administration, respectively.

Measurement of TVDT. The maximum tumor diameter was measured in the equilibrium phase of dynamic CT using electronic calipers. The baseline and follow-up CTs of the same patient were evaluated by the same examiner, who was blinded to the clinical patient data. The TVDT was calculated using the following equation $(11,12)$ :

$$
\text { TVDT }=(\mathrm{T} \log 2) / 3[\log (\mathrm{M} 1)-\log (\mathrm{M} 0)]
$$

where M0 is the first-time maximum diameter of the HCC, M1 is the second-time maximum diameter and $\mathrm{T}$ is the observation period. The differences in TVDT were compared based on the histological grade of HCC differentiation.

Measurement of T2AFP. The T2AFP was calculated using the following equation:

$$
\mathrm{T} 2 \mathrm{AFP}=(\mathrm{T} \log 2) /[\log (\mathrm{AFP} 1)-\log (\mathrm{AFP} 0)],
$$

where AFP0 is the first-time AFP level, AFP1 is the second-time AFP level and T is the observation period.

The AFP-L3 fraction level was measured simultaneously using a liquid-phase binding assay, with a result of $<10 \%$ being considered as negative and $\geq 10 \%$ as positive. The differences in T2AFP were compared among different histological grades and between AFP-L3-positive and -negative groups. Furthermore, the correlation between T2AFP and TVDT was analyzed.

Statistical analysis. The differences in tumor size and TVDT among histological grades of HCC differentiation were compared using the Kruskal-Wallis and Mann-Whitney U tests. The differences in T2AFP among histological grades of HCC differentiation were compared using the Mann-Whitney U test. The difference in T2AFP between AFP-L3-positive and -negative groups was compared using the Mann-Whitney U test. The Pearson correlation coefficient was used to evaluate the association between T2AFP and TVDT. For each statistical test, values of $\mathrm{P}<0.05$ were considered to indicate a statistically significant difference. Data were analyzed using SPSS for Windows, version 20.0J (SPSS Japan Inc., Tokyo, Japan).

\section{Results}

Correlation between TVDT and histological grade of HCC differentiation. To evaluate the grade of histological differentiation in each case, 22 nodules obtained from resected specimens and 31 nodules obtained by tumor biopsy were analyzed. The patient characteristics are summarized in Table I. The patient sample comprised 35 men and 18 women with a mean \pm SD age of $67.2 \pm 8.5$ years (range, $38-80$ years). The mean observation period was $45.7 \pm 25.4$ days (range, 14-114 days). The mean TVDT was 91.4 $\$ 93.6$ days (range, 
Table I. Characteristics of the patients included in the analysis of the correlation between tumor volume doubling time and histological grade of differentiation.

\begin{tabular}{lc}
\hline Characteristics & Values \\
\hline Age (years, mean \pm SD) & $67.2 \pm 8.5$ \\
Gender (no., male/female) & $35 / 18$ \\
Etiology (no., HBV/HCV/both negative) & $7 / 41 / 5$ \\
Fibrosis stage (no., F0/1/2/3/4) & $1 / 3 / 6 / 24 / 19$ \\
Child-Pugh class (no., A/B/C) & $37 / 15 / 1$ \\
Number of nodules (1/2/ $\geq 3$ ) & $30 / 12 / 11$ \\
Tumor size (mm, mean \pm SD) & $26.0 \pm 14.3$ \\
Histological differentiation (no., Ed1/2/3) & $13 / 28 / 12$ \\
AFP (ng/ml, mean \pm SD) & $1,149.6 \pm 2,255.2$ \\
AFP-L3 ( $\geq 10 \% /<10 \%)$ & $26 / 27$ \\
DCP (mAU/ml) & $4,222.5 \pm 3,759.6$ \\
\hline
\end{tabular}

$\mathrm{SD}$, standard deviation; HBV, hepatitis B virus; $\mathrm{HCV}$, hepatitis $\mathrm{C}$ virus; Ed, Edmondson grade; AFP, $\alpha$-fetoprotein; AFP-L3, Lens culinaris agglutinin-reactive fraction of $\alpha$-fetoprotein; DCP, des- $\gamma$ carboxyprothrombin.

Table II. Characteristics of patients included in the analysis of the correlation between $\alpha$-fetoprotein doubling time and histological grade of differentiation.

\begin{tabular}{lc}
\hline Characteristics & Values \\
\hline Age (years, mean \pm SD) & $67.5 \pm 8.2$ \\
Gender (no., male/female) & $27 / 17$ \\
Etiology (no., HBV/HCV/both negative) & $5 / 35 / 4$ \\
Fibrosis stage (no., F0/1/2/3/4) & $3 / 2 / 5 / 18 / 16$ \\
Child-Pugh class (no., A/B/C) & $33 / 11 / 0$ \\
Number of nodules $(1 / 2 / \geq 3)$ & $30 / 12 / 11$ \\
Tumor size (mm, mean $\pm \mathrm{SD})$ & $36.3 \pm 21.2$ \\
Histological differentiation (no., Ed1/2/3) & $0 / 29 / 15$ \\
AFP (ng/ml, mean $\pm \mathrm{SD})$ & $7,695.0 \pm 33,450.7$ \\
AFP-L3 ( $\geq 10 \% /<10 \%)$ & $34 / 10$ \\
DCP (mAU/ml) & $11,053.0 \pm 23,973.8$
\end{tabular}

$\mathrm{SD}$, standard deviation; $\mathrm{HBV}$, hepatitis $\mathrm{B}$ virus; $\mathrm{HCV}$, hepatitis $\mathrm{C}$ virus; Ed, Edmondson grade; AFP, $\alpha$-fetoprotein; AFP-L3, Lens culinaris agglutinin-reactive fraction of $\alpha$-fetoprotein; DCP, des- $\gamma$ carboxyprothrombin.

8.7-406.3 days). Histologically, 13 nodules were classified as Edmondson grade 1 (Ed1), 28 were Ed2 and 12 were Ed3. The mean tumor diameter \pm SD of all the HCCs, Ed1, Ed 2 and Ed3 was 25.6 $\pm 14.3,20.0 \pm 8.0,30.6 \pm 14.8$ and $21.5 \pm 9.2 \mathrm{~mm}$, respectively, exhibiting no statistically significant differences among different histological grades $(\mathrm{P}=0.066)$. No significant correlation was observed between TVDT and tumor diameter (correlation coefficient, $-0.06 ; \mathrm{P}=0.668$ ). The mean TVDT \pm SD for Ed1, Ed2 and Ed3 was 138.3 $\pm 110.3,94.9 \pm 91.5$ and $32.2 \pm 20.8$ days, respectively. The TVDT was significantly

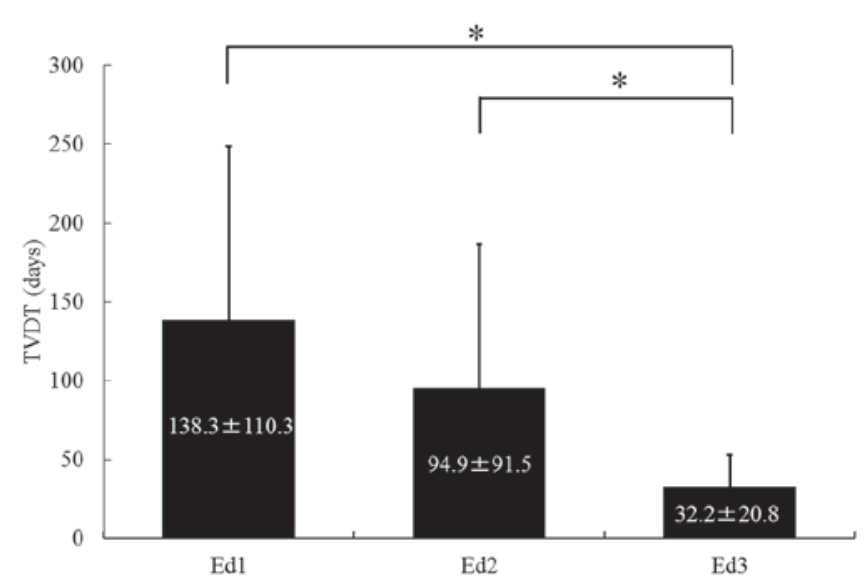

Figure 1. Tumor volume doubling time (TVDT) according to histological grade of differentiation of hepatocellular carcinoma. The mean \pm standard deviation TVDT for Edmondson grade 1 (Ed1), Ed2 and Ed3 was $138.3 \pm 110.3,94.9 \pm 91.5$ and $32.2 \pm 20.8$ days, respectively. The TVDT was significantly longer for Ed1 and Ed2 compared to that for $\mathrm{Ed} 3$ ("P<0.05).

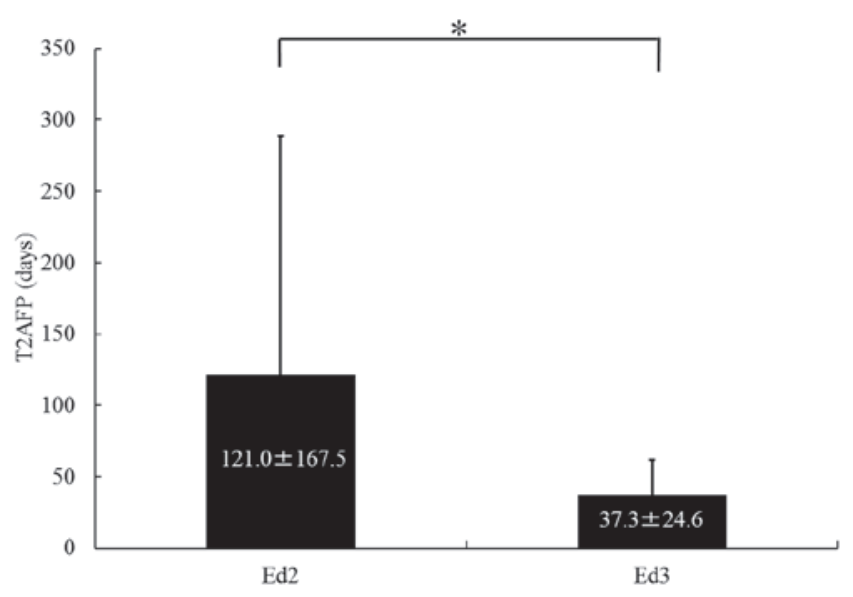

Figure 2. Serum $\alpha$-fetoprotein doubling time (T2AFP) according to histological grade of differentiation of hepatocellular carcinoma. The mean \pm standard deviation T2AFP for Edmondson grade $2(E d 2)$ and Ed3 was $121.0 \pm 167.5$ and $37.3 \pm 24.6$ days, respectively. There was a statistically significant difference in T2AFP between Ed2 and Ed3 $\left({ }^{*} \mathrm{P}<0.01\right)$.

longer for Ed1 and Ed2 compared to that for Ed3 $(\mathrm{P}<0.05)$, although no significant difference in TVDT was observed between Ed1 and Ed2 (P=0.11) (Fig. 1).

Correlation between T2AFP and histological grade of HCC differentiation. In order to evaluate the grade of histological differentiation, 17 nodules obtained from resected specimens and 27 nodules obtained by tumor biopsy were analyzed. The patient characteristics are summarized in Table II. The patient sample comprised 27 men and 17 women with a mean age $\pm \mathrm{SD}$ of $67.5 \pm 8.2$ years (range, $48-86$ years). The mean \pm SD examination interval was $38.4 \pm 21.9$ days (range, 14-113 days). No Ed1 HCC exhibited serum baseline AFP levels of $>200 \mathrm{ng} / \mathrm{ml}$. A total of 29 nodules were $\mathrm{Ed} 2$ and 15 were Ed3. The mean \pm SD T2AFP was $121.0 \pm 167.5$ and $37.3 \pm 24.6$ days for $E d 2$ and $E d 3$, respectively. There was a statistically significant difference in T2AFP between Ed2 and Ed3 (P<0.01) (Fig. 2). 


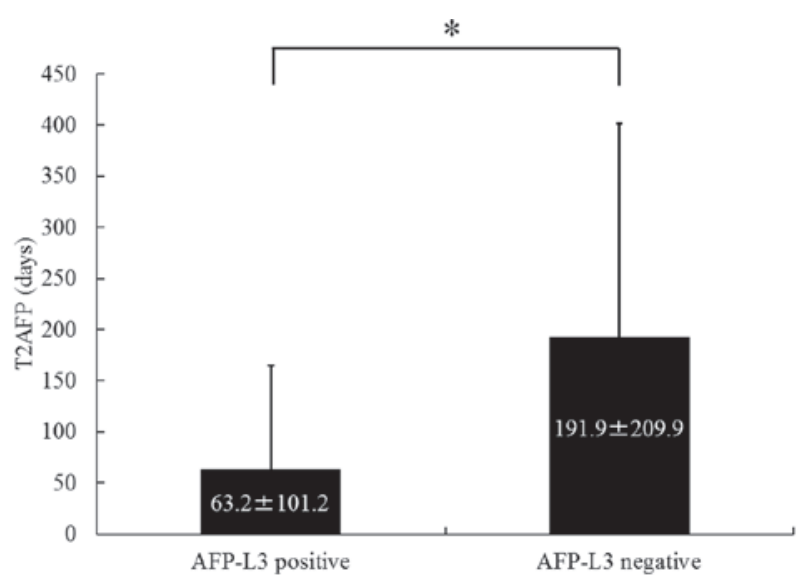

Figure 3. Serum $\alpha$-fetoprotein doubling time (T2AFP) according to positivity for Lens culinaris agglutinin-reactive fraction of $\alpha$-fetoprotein (AFP-L3). Using an AFP-L3 level of $>10 \%$ as positive, the mean \pm standard deviation T2AFP in the AFP-L3-positive and -negative groups was 63.2 \pm 101.2 and $191.9 \pm 209.9$ days, respectively. T2AFP was significantly shorter in the AFP-L3-positive compared to that in the AFP-L3-negative group $\left({ }^{*} \mathrm{P}<0.01\right)$.

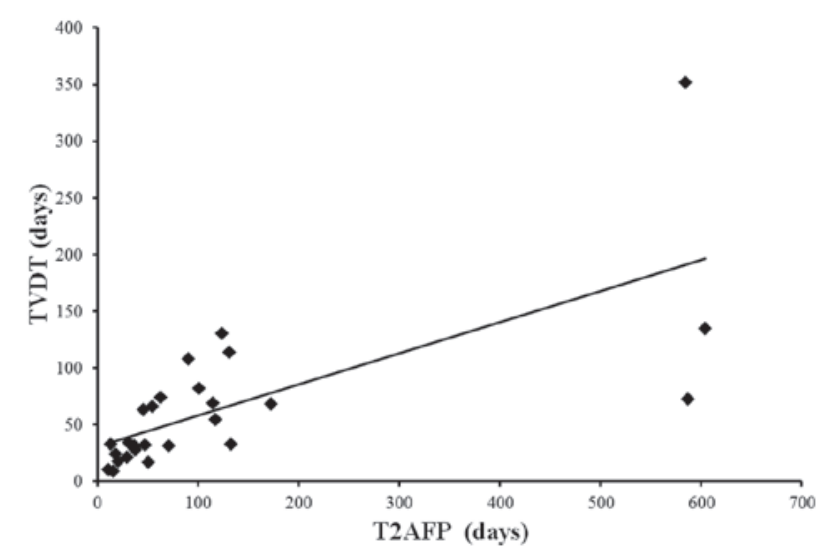

Figure 4. Correlation between tumor volume doubling time (TVDT) and serum $\alpha$-fetoprotein doubling time (T2AFP). A strong and significant correlation was observed between the two (correlation coefficient, $0.70 ; \mathrm{P}<0.01$ ).

Correlation between T2AFP and AFP-L3. A total of 34 patients were AFP-L3-positive and 10 were AFP-L3-negative. The mean \pm SD T2AFP was $63.2 \pm 101.2$ days for AFP-L3-positive patients and 191.9 \pm 209.9 days for AFP-L3-negative patients. The T2AFP was significantly shorter in the AFP-L3-positive group compared to that in the AFP-L3-negative group $(\mathrm{P}<0.01)$ (Fig. 3).

Correlation between T2AFP and TVDT. The T2AFP was calculated for 27 of the 44 cases and an analysis was performed. A strong and significant correlation was identified between T2AFP and TVDT (correlation coefficient, 0.70; P<0.01) (Fig. 4).

\section{Discussion}

Previous studies on the growth rate of HCC revealed a wide range of TVDTs, from 13.5 to 2,671 days (13-17). Ebara et al (18) suggested that the growth rate of HCC is not always consistent, even in the same patient, and may change during the course of HCC progression. Sheu et al (19) indicated that TVDT in HCC was positively correlated with initial tumor size. By contrast, other studies demonstrated no significant correlation between TVDT and tumor size $(14,15,20,21)$. A previous study by Kubota et al (15) evaluated small HCCs $(<20 \mathrm{~mm})$ and demonstrated that the TVDT ranged widely, from 34.8 to 496.4 days. The present results indicated a similar range of the TVDTs, from 8.7 to 343.9 days. Furthermore, no significant correlation was observed between TVDT and tumor diameter. These findings strongly suggest that rapidly growing HCCs with high-grade malignant potential may be present even among small HCCs.

The wide range of TVDTs in small HCCs may be explained by differences in the histological grade of differentiation in each nodule. Although Sheu et al (19) observed that TVDT was not correlated with histological type or grade of malignancy, a significant correlation between TVDT and histological differentiation was demonstrated by some studies $(18,21)$. Namely, slowly growing HCCs tend to be histologically well-differentiated, whereas rapidly growing HCCs tend to be moderately or poorly differentiated. In the present study, TVDT was significantly shorter for poorly differentiated compared to that for either wellor moderately differentiated HCCs and was the shortest among the three histological grades of HCC differentiation. Thus, the TVDT of each HCC appears to be significantly affected by the histological grade of differentiation.

Ebara et al (18) and Okazaki et al (20) identified TVDT as one of the factors affecting survival in HCC patients. Furthermore, Cucchetti et al (21) reported that TVDT predicts the recurrence of single HCCs after surgical resection. Those results demonstrated that HCCs with vascular invasion, poor or undifferentiation on histological examination and serum AFP levels $>200 \mathrm{ng} / \mathrm{ml}$ exhibited faster tumor growth, unrelated to tumor size. Thus, the TVDT of HCC reflects the histological grade of differentiation or the grade of malignancy in the process of cancer progression and the assessment of TVDT is considered crucial for deciding the treatment of HCC patients, particularly those with small HCCs treatable by percutaneous local ablation or surgical resection.

However, in order to calculate TVDT, an obvious increase in tumor size must be evident from a comparison of two consecutive imaging examinations. If a close association exists between TVDT and a tumor marker doubling time, that particular tumor marker doubling time may serve as a surrogate for TVDT. Sheu et al (19) reported that, although no significant correlation was observed between TVDT and initial serum AFP levels, TVDT was closely correlated with T2AFP. Our results were consistent with the previous findings. Furthermore, T2AFP was correlated with histological grade of differentiation and the level of AFP-L3, which is another indicator of the grade of malignancy in HCC. Masuda et al (22) reported that the doubling times of preoperative serum AFP or des- $\gamma$ carboxyprothrombin may be useful predictors of recurrence and prognosis. Those results together with ours, strongly indicate that T2AFP represents a valuable serological index reflecting the malignant potential of HCC. Since the assessment of T2AFP is easier, simpler and faster compared to that of TVDT, T2AFP appears to offer a more useful tool for evaluating tumor growth rates compared to TVDT.

Certain limitations must be considered when interpreting the results of the present study. First, this study on HCC growth rate was small and retrospective. A prospective study would be preferable; however, such a study is not ethically feasible, since 
treatment would have to be delayed from the day of diagnosis until confirmation of tumor enlargement. For this reason, the majority of previous studies involved retrospective investigations of a relatively small number of subjects. Second, the histological grade of differentiation was assessed using not only resected specimens, but also tumor biopsy specimens. Since the histological differentiation of HCC is often heterogeneous, the assessment of the histological grade of differentiation may not reflect the least differentiated histology in the tumor. However, the present study strongly demonstrated that TVDT was significantly correlated with histological grade of differentiation, consistent with previous studies $(18,21)$. Third, since the tumor size of massive or diffuse-type HCC cannot be accurately measured, the analysis of TVDT in the present study was limited to nodular-type HCC. Thus, the present results cannot be generalized, although the possibility that T2AFP reflects tumor growth rates even in massive or diffuse-type HCC requires further investigation. Fourth, T2AFP cannot be used to assess the tumor growth rate when $\mathrm{HCC}$ is not detected on imaging. In the present study, T2AFP was analyzed only in HCC patients with baseline serum AFP levels of $>200 \mathrm{ng} / \mathrm{ml}$ that remained higher thereafter, to allow the exclusion of non-specific AFP elevations (8). However, patients with AFP > $200 \mathrm{ng} / \mathrm{ml}$ without imaging evidence of $\mathrm{HCC}$ may be a frequent finding. Furthermore, the assessment of tumor growth rate using T2AFP is not possible for non-AFP-producing HCCs. Furthermore, tumor growth rates cannot be assessed when AFP-producing activity is low and T2AFP is long. However, since the HCCs for which T2AFP could be assessed in the present study were all moderately or poorly differentiated, T2AFP may be considered useful for identifying HCCs with a high-grade malignant potential. Finally, the interval used for T2AFP measurements in the present analysis varied greatly (range, 14-113 days). It was previously reported that the growth rate of HCC is not always consistent and the increases in the serum AFP levels are also not always linear (18). In addition, dedifferentiation of HCC is often observed during growth. Thus, the optimal interval between two AFP measurements has not been determined.

In conclusion, a significant correlation was observed among serological indices, histological grade of differentiation and TVDT, but not tumor size. The TVDT and T2AFP were shorter with advancing histological grade of differentiation, or grade of malignancy. The T2AFP was shorter in the AFP-L3-positive compared to that in the AFP-L3-negative group. A strong correlation was observed between TVDT and T2AFP; therefore, when deciding the treatment for HCC patients, attention should be paid to a short T2AFP and/or AFP-L3 positivity, since poorly differentiated HCC is more likely to exhibit microscopic vascular invasion or intrahepatic metastases.

\section{Acknowledgements}

The authors would like to thank Ms. Kazu Konishi for her excellent secretarial assistance. This study was supported in part by a grant-in-aid from Takeda Pharmaceutical Company Limited.

\section{References}

1. Theise ND: Macroregenerative (dysplastic) nodules and hepatocarcinogenesis: theoretical and clinical considerations. Semin Liver Dis 15: 360-371, 1995.
2. Fukuda S, Itamoto T, Nakahara H, et al: Clinicopathologic features and prognostic factors of resected solitary small-sized hepatocellular carcinoma. Hepatogastroenterology 52: 1163-1167, 2005.

3. Sasaki Y, Imaoka S, Ishiguro S, et al: Clinical features of small hepatocellular carcinomas as assessed by histologic grades. Surgery 119: 252-260, 1996.

4. Oishi K, Itamoto T, Amano H, et al: Clinicopathologic features of poorly differentiated hepatocellular carcinoma. J Surg Oncol 95: 311-316, 2007.

5. Yamashita F, Tanaka M, Satomura S and Tanikawa K: Prognostic significance of Lens culinaris agglutinin A-reactive alpha-fetoprotein in small hepatocellular carcinomas. Gastroenterology 111: 996-1001, 1996.

6. Tada T, Kumada T, Toyoda H, et al: Relationship between Lens culinaris agglutinin-reactive alpha-fetoprotein and pathologic features of hepatocellular carcinoma. Liver Int 25: 848-853, 2005.

7. Miyaaki H, Nakashima O, Kurogi M, Eguchi K and Kojiro M: Lens culinaris agglutinin-reactive alpha-fetoprotein and protein induced by vitamin $\mathrm{K}$ absence II are potential indicators of a poor prognosis: a histopathological study of surgically resected hepatocellular carcinoma. J Gastroenterol 42: 962-968, 2007.

8. Ebara M, Ohto M, Shinagawa T, et al: Natural history of minute hepatocellular carcinoma smaller than three centimeters complicating cirrhosis. A study in 22 patients. Gastroenterology 90 : 289-298, 1986.

9. Edmondson HA and Steiner PE: Primary carcinoma of the liver: a study of 100 cases among 48,900 necropsies. Cancer 7: 462-503, 1954.

10. Bedossa $P$ and Poynard T: An algorithm for the grading of activity in chronic hepatitis C. The METAVIR Cooperative Study Group. Hepatology 24: 289-293, 1996.

11. Collins VP, Loeffler RK and Tivey H: Observations on growth rates of human tumors. Am J Roentgenol Radium Ther Nucl Med 76: 988-1000, 1956.

12. Schwartz M: A biomathematical approach to clinical tumor growth. Cancer 14: 1272-1294, 1961.

13. Bolondi L, Benzi G, Santi V, et al: Relationship between alpha-fetoprotein serum levels, tumour volume and growth rate of hepatocellular carcinoma in a western population. Ital J Gastroenterol 22: 190-194, 1990

14. Barbara L, Benzi G, Gaiani S, et al: Natural history of small untreated hepatocellular carcinoma in cirrhosis: a multivariate analysis of prognostic factors of tumor growth rate and patient survival. Hepatology 16: 132-137, 1992.

15. Kubota K, Ina H, Okada Y and Irie T: Growth rate of primary single hepatocellular carcinoma: determining optimal screening interval with contrast enhanced computed tomography. Dig Dis Sci 48: 581-586, 2003.

16. Taouli B, Goh JS, Lu Y, et al: Growth rate of hepatocellular carcinoma: evaluation with serial computed tomography or magnetic resonance imaging. J Comput Assist Tomogr 29: 425-429, 2005.

17. Furlan A, Marin D, Agnello F, et al: Hepatocellular carcinoma presenting at contrast-enhanced multi-detector-row computed tomography or gadolinium-enhanced magnetic resonance imaging as a small $(\leq 2 \mathrm{~cm})$, indeterminate nodule: growth rate and optimal interval time for imaging follow-up. J Comput Assist Tomogr 36: 20-25, 2012.

18. Ebara M, Hatano R, Fukuda H, Yoshikawa M, Sugiura N and Saisho H: Natural course of small hepatocellular carcinoma with underlying cirrhosis. A study of 30 patients. Hepatogastroenterology 45 (Suppl 3): 1214-1220, 1998.

19. Sheu JC, Sung JL, Chen DS, et al: Growth rate of asymptomatic hepatocellular carcinoma and its clinical implications. Gastroenterology 89: 259-266, 1985.

20. Okazaki N, Yoshino M, Yoshida T, et al: Evaluation of the prognosis for small hepatocellular carcinoma based on tumor volume doubling time. A preliminary report. Cancer 63: 2207-2210, 1989

21. Cucchetti A, Vivarelli M, Piscaglia F, et al: Tumor doubling time predicts recurrence after surgery and describes the histological pattern of hepatocellular carcinoma on cirrhosis. J Hepatol 43: 310-316, 2005.

22. Masuda T, Beppu T, Horino K, et al: Preoperative tumor marker doubling time is a useful predictor of recurrence and prognosis after hepatic resection of hepatocellular carcinoma. J Surg Oncol 102: 490-496, 2010. 